Andrea Valeria Arrossi, MD

Department of Pathology, Cleveland Clinic
Carol Farver, MD

Department of Pathology, University of Michigan,

Ann Arbor, Michigan

\title{
The pulmonary pathology of COVID-19
}

\section{Posted September 17, 2020}

\section{ABSTRACT}

A growing number of international postmortem studies identify acute and organizing diffuse alveolar damage (DAD) as the main pathologic feature of lung injury in patients with COVID-19. Other forms of acute lung injury, including organizing pneumonia, and acute fibrinous and organizing pneumonia are seen. Acute neutrophilic infiltrates have been observed, most frequently as the manifestation of a superimposed bacterial pneumonia. SARS-CoV-2 has been detected in type I and type II pneumocytes and bronchial epithelial cells using electron microscopy, immunohistochemistry, and in situ hybridization, and likewise, viral transcripts were localized with RNA probes in pneumocytes. However, the presence of true viral cytopathic effect seen with light microscopy remains to be defined. Interestingly, vascular changes are frequently observed in association with $D A D$, which include severe endothelial injury/endothelialitis, hemorrhage, and thrombotic and microangiopathic vasculopathy. Since similar vascular changes also occur in cases of DAD independent of the etiology, whether the vascular pathology in COVID lungs has unique features and represents a separate pathologic process is under investigation.

\section{INTRODUCTION}

Corona virus disease (COVID-19), caused by the novel coronavirus SARS-CoV-2, has spread globally since the initial outbreak in China in late 2019. On March 11, 2020, the infection was declared a pandemic by the World Health Organization. Patients infected with the virus may be asymptomatic or have symptoms with varying degrees of severity involving predominantly the respiratory tract. About 20\% of patients develop severe disease and the mortality rate of patients with COVID-19 is .5\% to 2\%. Research on all aspects of the disease has been fast moving as understanding the epidemiology, pathogenesis, clini$\mathrm{cal}$, and pathologic characteristics of SARS-CoV-2 infection are crucial for the development of immuni-

The statements and opinions expressed in COVID-19 Curbside Consults are based on experience and the available literature as of the date posted. While we try to regularly update this content, any offered recommendations cannot be substituted for the clinical judgment of clinicians caring for individual patients.

doi:10.3949/ccjm.87a.ccc063 zation and treatment strategies.

Studies of the histopathologic changes of COVID19 are predominately conducted postmortem after clinically severe forms of the disease. The novelty and high infectivity of COVID-19 requires extra biosafety measures, including appropriate personal and environmental protective equipment. Because of this, many pathology laboratories have limited autopsies in these patients. Nonetheless, numerous published studies of the histopathology of COVID19 have emerged and increased recently, resulting in a growing consensus about the main features of the pulmonary pathology of COVID-19.

\section{HISTOPATHOLOGY}

The predominant pathology in the lungs of deceased patients with COVID-19 is diffuse alveolar damage (DAD) ${ }^{1-14}$ This pattern of acute lung injury is the histologic finding observed in patients with acute respiratory distress syndrome regardless of the etiology. It results from epithelial and endothelial injury after an initial insult that leads to increased permeability of the alveolar-capillary barrier with consequent edema, release of proinflammatory and repair chemical mediators, impairment of the metabolism of surfactant, and abnormalities in the coagulation pathways. The interplay of these mechanisms varies depending on the causative agent, but the ultimate morphology of DAD has similar features. DAD is divided into 2 main phases, the acute phase and the organizing phase. ${ }^{15}$ Microscopically, the acute phase consists of vascular congestion with alveolar septal edema, fibrinoid exudates within the alveolar spaces and hyaline membranes. In the organizing phase of DAD, the alveolar interstitium is replaced by fibroblastic tissue, which can be prominent, and the alveolar septae are lined by reactive/reparative type II pneumocyte hyperplasia.

The pulmonary pathology findings of COVID19 have been reported predominantly from autopsy cases of severely ill patients with acute respiratory distress syndrome, many of them with long hospital stays before expiration. Accordingly, DAD (Figure 1 ), in varying stages of evolution, is the predominant pathology pattern, though patterns of other forms 
of acute lung injury including acute fibrinous and organizing pneumonia, ${ }^{4,6,10}$ and organizing pneumonia $^{2,6,9,13}$ may occur. Acute neutrophilic infiltrates have been observed in some cases, most frequently as the manifestation of a superimposed bacterial pneumonia. Large airway inflammatory changes include mucosal ulceration, acute and/or chronic inflammation. A recent study observed that $45 \%$ $(17 / 38)$ of patients who were never intubated and $52 \%$ of patients with no associated bacterial or fungal pneumonia showed acute inflammation of the large airways. The authors did not find a significant relationship between overall large airway inflammation and intubation, or bacterial or fungal pneumonia. ${ }^{13}$

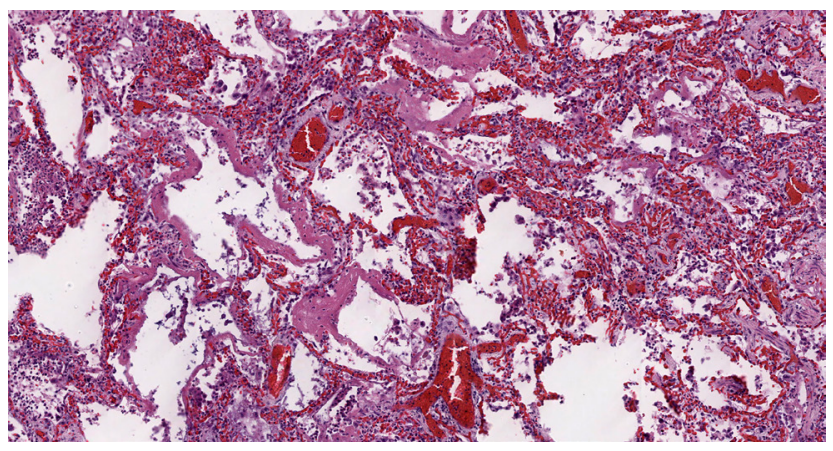

Figure 1. Acute diffuse alveolar damage: scattered hyaline membranes line the alveolar walls with vascular congestion and interstitial inflammation present within the interstitium (Hematoxylin and eosin; 200x).

In addition to DAD, other findings described by pathologists include interstitial lymphocyte-predominant mononuclear cell infiltrates $3,8,10,16,17$ and vascular changes such as severe endothelial injury/endothelialitis, ${ }^{10,16,18,19}$ thrombotic and microangiopathic vasculopathy $2,4-6,9,10,13,16,18-20$ (Figure 2), and pulmonary hemorrhage. . $^{3-11}$ The microangiopathy and coagulopathy in patients with COVID-19 is thought to result from the endothelial cell activation and damage occurring secondary to the binding of the virus to the endothelial cells via angiotensin-converting enzyme 2 receptors, as well as activation of the coagulation through several procoagulant pathways induced by a dysregulated immune response or cytokine storm that occur in severe forms of the disease. ${ }^{18,21,22}$ However, these events can occur in DAD independent of the cause, thus the significance of their presence in COVID-19 lungs has yet to be elucidated. Furthermore, a recent postmortem histologic examination of DAD in the lungs of patients with COVID-19 compared with patients with DAD from other causes revealed that DAD is histologically indistinguishable in the 2 groups. ${ }^{4}$

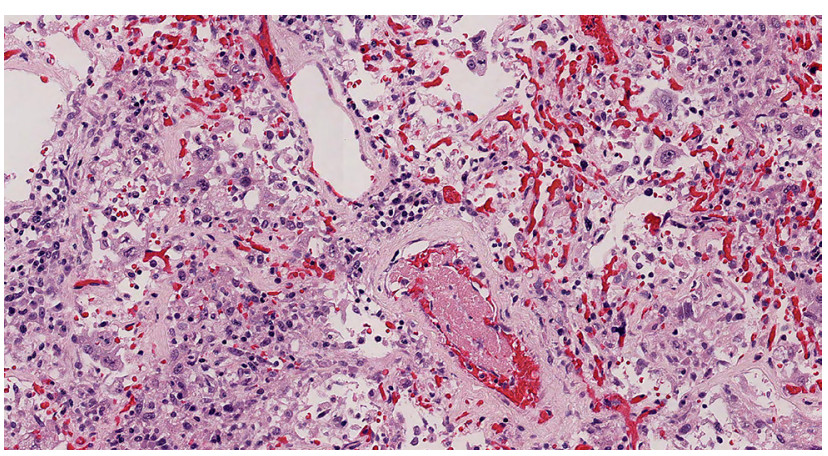

Figure 2. Organizing thrombus: an early organizing thrombus is present within a small artery. The surrounding lung reveals areas of vascular congestion (Hematoxylin and eosin; 200x)

Other histologic features reported in lungs infected with SARS-CoV-2 include intra-alveolar hemorrhage with both hemosiderin and intra-alveolar fibrin present. The etiology of this hemorrhage is not clear, though microvascular fragility due to complement activation has been speculated. Also, many studies report the presence of atypical large pneumocytes or atypical multinucleated giant cells and have suggested that the changes are due to viral cytopathic effect. In this regard, the virus has been detected in type I and type II pneumocytes using electron microscopy, immunohistochemistry and in situ hybridization, and RNA probes have localized viral transcripts in pneumocytes. ${ }^{1,6,9,12-14,23-27}$ However, specific morphologic features of a viral cytopathic effect in the lungs of patients with COVID-19 using light microscopy remain to be defined.

The discrepancies in the reported pulmonary pathology of COVID-19 are often based on the determination of DAD or other causes. The pathological definition of DAD can vary among authors. Since DAD can have lymphocytic infiltrates early in the disease with congested capillaries, especially when a virus is the etiology, the reports of a separate capillaritis-like pathology may arise from cases of a very early DAD where this pattern can be seen. Similarly, as stated, thrombosis that is seen throughout these lungs, is also a feature of DAD. However, the controversy arises from the extent of the thrombi seen in COVID-19, that is, the number of thrombi in COVID-19 lungs beyond what would manifest in DAD due to other causes. Most recently, studies that have quantitated the amount of thrombi present, sug- 
gest that the number present is beyond what would be seen in a non-COVID-19 DAD and most likely represent a thrombosis that is a separate pathologic process from DAD. ${ }^{14,16}$

\section{FINAL COMMENTS}

A growing number of international postmortem studies identify acute DAD as the main pathologic feature of lung injury in patients with COVID-19. Other forms of acute lung injury, including acute fibrinous and organizing pneumonia, and organizing $\mathrm{DAD}$ are seen, as are numerous thrombi and areas of pulmonary hemorrhage. Though multinucleated giant cells and enlarge pneumocytes with cleared chromatin are found, whether these represent true viral cytopathic effects remains to be defined.

\section{REFERENCES}

1. Bradley BT, Maioli H, Johnston R, et al. Histopathology and ultrastructural findings of fatal COVID-19 infections in Washington State: a case series [published correction appears in Lancet. 2020 Aug 1;396(10247):312]. Lancet. 2020;396(10247):320-332. doi:10.1016/ S0140-6736(20)31305-2

2. Carsana L, Sonzogni A, Nasr A, et al. Pulmonary post-mortem findings in a series of COVID-19 cases from northern Italy: a twocentre descriptive study [published online ahead of print, 2020 Jun 8]. Lancet Infect Dis. 2020;S1473-3099(20)30434-5. doi:10.1016/ S1473-3099(20)30434-5

3. Fox SE, Akmatbekov A, Harbert JL, Li G, Quincy Brown J, Vander Heide RS. Pulmonary and cardiac pathology in African American patients with COVID-19: an autopsy series from New Orleans. Lancet Respir Med. 2020;8(7):681-686. doi:10.1016/\$2213-2600(20)30243-5

4. Konopka KE, Nguyen T, Jentzen JM, et al. Diffuse alveolar damage (DAD) resulting from coronavirus disease 2019 Infection is Morphologically Indistinguishable from Other Causes of DAD [published online ahead of print, 2020 Jun 15]. Histopathology. 2020;10.1111/ his. 14180. doi: $10.1111 /$ his. 14180

5. Menter T, Haslbauer JD, Nienhold R, et al. Postmortem examination of COVID-19 patients reveals diffuse alveolar damage with severe capillary congestion and variegated findings in lungs and other organs suggesting vascular dysfunction [published online ahead of print, 2020 May 4]. Histopathology. 2020;10.1111/his.14134. doi:10.1111/his. 14134

6. Polak SB, Van Gool IC, Cohen D, von der Thüsen JH, van Paassen J. A systematic review of pathological findings in COVID-19: a pathophysiological timeline and possible mechanisms of disease progression [published online ahead of print, 2020 Jun 22]. Mod Pathol. 2020;1-11. doi:10.1038/s41379-020-0603-3

7. Schaller T, Hirschbühl K, Burkhardt $K$, et al. Postmortem Examination of Patients With COVID-19. JAMA. 2020;323(24):2518-2520. doi:10.1001/jama.2020.8907

8. Xu Z, Shi L, Wang Y, et al. Pathological findings of COVID-19 associated with acute respiratory distress syndrome [published correction appears in Lancet Respir Med. 2020 Feb 25;:]. Lancet Respir Med. 2020;8(4):420-422. doi:10.1016/S2213-2600(20)30076-X

9. Sauter JL, Baine MK, Butnor KJ, et al. Insights into pathogenesis of fatal COVID-19 pneumonia from histopathology with immunohistochemical and viral RNA studies [published online ahead of print, $2020 \mathrm{Jul}$ 2]. Histopathology. 2020;10.1111/his.14201. doi:10.1111/ his. 14201

10. von der Thüsen J, van der Eerden M. Histopathology and genetic susceptibility in COVID-19 pneumonia [published online ahead of print, 2020 Apr 30]. Eur J Clin Invest. 2020;e13259. doi:10.1111/ eci. 13259

11. Tian S, Xiong Y, Liu H, et al. Pathological study of the 2019 novel coronavirus disease (COVID-19) through postmortem core biopsies. Mod Pathol. 2020;33(6):1007-1014. doi:10.1038/s41379-020-0536-x

12. Martines RB, Ritter JM, Matkovic E, et al. Pathology and Pathogenesis of SARS-CoV-2 Associated with Fatal Coronavirus Disease, United States. Emerg Infect Dis. 2020;26(9):2005-2015. doi:10.3201/ eid2609.202095

13. Borczuk AC, Salvatore SP, Seshan SV, et al. COVID-19 pulmonary pathology: a multi-institutional autopsy cohort from Italy and New York City [published online ahead of print, 2020 Sep 2]. Mod Pathol. 2020;10.1038/s41379-020-00661-1. doi:10.1038/s41379-020-00661-1

14. Hanley B, Naresh KN, Roufosse C, et al. Histopathological findings and viral tropism in UK patients with severe fatal COVID-19: a post-mortem study [published online ahead of print, 2020 Aug 20]. Lancet Microbe. 2020;10.1016/S2666-5247(20)30115-4. doi:10.1016/ S2666-5247(20)30115-4

15. Beasley MB. Acute lung injury. In: Tomashefski, Jr. JF, Cagle PT, Farver CF, Fraire AE, eds. Dail and Hammar's Pulmonary Pathology Volume 1 Nonneoplastic Lung Disease. 3rd ed. Springer; 2008: 64-83

16. Ackermann M, Verleden SE, Kuehnel M, et al. Pulmonary Vascular Endothelialitis, Thrombosis, and Angiogenesis in Covid-19. N Engl J Med. 2020;383(2):120-128. doi:10.1056/NEJMoa2015432

17. Konopka KE, Wilson A, Myers JL. Postmortem Lung Findings in a Patient With Asthma and Coronavirus Disease 2019. Chest. 2020;158(3):e99-e101. doi:10.1016/j.chest.2020.04.032

18. Varga Z, Flammer AJ, Steiger $\mathbf{P}$, et al. Endothelial cell infection and endotheliitis in COVID-19. Lancet. 2020;395(10234):1417-1418. doi:10.1016/50140-6736(20)30937-5

19. Wichmann D, Sperhake JP, Lütgehetmann M, et al. Autopsy Findings and Venous Thromboembolism in Patients With COVID-19: A Prospective Cohort Study. Ann Intern Med. 2020;173(4):268-277. doi:10.7326/M20-2003

20. Lax SF, Skok K, Zechner $P$, et al. Pulmonary Arterial Thrombosis in COVID-19 With Fatal Outcome : Results From a Prospective, Single-Center, Clinicopathologic Case Series. Ann Intern Med. 2020;173(5):350-361. doi:10.7326/M20-2566

21. Connors JM, Levy JH. COVID-19 and its implications for thrombosis and anticoagulation. Blood. 2020;135(23):2033-2040. doi:10.1182/ blood. 2020006000

22. Zhu Z, Cai T, Fan L, et al. Clinical value of immune-inflammatory parameters to assess the severity of coronavirus disease 2019. Int J Infect Dis. 2020;95:332-339. doi:10.1016/j.ijid.2020.04.041

23. Best Rocha A, Stroberg E, Barton LM, et al. Detection of SARS-CoV-2 in formalin-fixed paraffin-embedded tissue sections using commercially available reagents [published online ahead of print, $2020 \mathrm{Jul}$ 9]. Lab Invest. 2020;1-5. doi:10.1038/s41374-020-0464-x

24. Schaefer IM, Padera RF, Solomon IH, et al. In situ detection of SARS-CoV-2 in lungs and airways of patients with COVID-19 [published online ahead of print, 2020 Jun 19]. Mod Pathol. 2020;1-11. doi:10.1038/s41379-020-0595-z

25. Sekulic M, Harper H, Nezami BG, et al. Molecular Detection of SARS-CoV-2 Infection in FFPE Samples and Histopathologic Findings in Fatal SARS-CoV-2 Cases. Am J Clin Pathol. 2020;154(2):190-200. doi:10.1093/ajcp/aqaa091

26. Zhang H, Zhou P, Wei Y, et al. Histopathologic Changes and SARSCoV-2 Immunostaining in the Lung of a Patient With COVID-19. Ann Intern Med. 2020;172(9):629-632. doi:10.7326/M20-0533

27. Liu J, Babka AM, Kearney BJ, Radoshitzky SR, Kuhn JH, Zeng X. Molecular detection of SARS-CoV-2 in formalin-fixed, paraffinembedded specimens. JCI Insight. 2020;5(12):e139042. Published 2020 Jun 18. doi:10.1172/jci.insight. 139042

Correspondence: Andrea Valeria Arrossi, MD, Department of Pathology, L25, Cleveland Clinic, 9500 Euclid Avenue, Cleveland, $\mathrm{OH}$ 44195; arrossa@ccf.org 